\title{
Evaluation of Oxidant and Anti-Oxidant Activity in Rheumatoid Arthritis Patients and Their Effects on Rheumatoid Arthritis Disease Activity
}

\section{Taraneh Dormohammadi Toosi}

Tehran University of Medical Sciences

Abbas Dehghani

Tehran University of Medical Sciences

Ramin Rezaei

Tehran University of Medical Sciences

Mohammad Hossein Asgardoon

Tehran University of Medical Sciences

Hossein Mirmiranpour ( $\nabla$ h_mirmiranpoor@yahoo.com )

Tehran University of Medical Sciences

\section{Abdolrahman Rostamian}

Iran University of Medical Sciences School of Behavioral Sciences and Mental Health

\section{Safieh Movasseghi}

Tehran University of Medical Sciences

\section{Shaghayegh Pezeshki}

Iran University of Medical Sciences School of Behavioral Sciences and Mental Health

\section{Payam Hashemi}

Tehran University of Medical Sciences

\section{Alireza Esteghamati}

Tehran University of Medical Sciences

\section{Research article}

Keywords: rheumatoid arthritis, antioxidant, oxidant, disease activity, DAS28

Posted Date: January 13th, 2020

DOI: https://doi.org/10.21203/rs.2.20677/v1

License: (c) (1) This work is licensed under a Creative Commons Attribution 4.0 International License.

Read Full License 


\section{Abstract}

\section{Background}

Rheumatoid arthritis (RA) is the most common cause of systemic inflammatory arthritis which causes joint destruction. The pathogenesis of RA is not fully understood, but it seems imbalance between oxidant and antioxidant process, plays a significant role on it. As a result, suppression of these mechanisms would be helpful to subside inflammation and eventually control of the disease activity. The aim of this study was to evaluate the level of oxidant and antioxidant and their effects on disease activity in RA patients.

\section{Methods}

In the following case-control study, we evaluated the levels of Malondialdehyde (MDA) and oxidized LowDensity Lipoproteins (ox.LDL) as oxidative factors and Catalase (CAT), Glutathione Peroxide (GSH-Px) and Superoxide Dismutase (SOD) as antioxidants. Also tumor Necrosis Factor (TNF)-a, Interleukin (IL) $1 \beta$ and IL6 were measured as inflammatory factors.

\section{Results}

43 RA patients and 43 healthy people were enrolled. Significant differences were found in the average levels of MDA, ox.LDL, CAT, GPX, SOD, TNFa, IL1 beta and IL6 between two groups ( $p$-value < 0.001), but we did not find any significant differences between two groups of patients based on DAS28 (p-value> $0.05)$.

\section{Conclusion}

The results of our study showed that there were increased oxidative activities in RA patients in comparison to the control group which indicated the presence of inflammatory process causing cellular damage in the patients group. As a consequence, adding some antioxidant agents to RA treatment might have some advantages for the disease control. Based on our findings, it seems that the oxidative process did not have any effect on the disease severity. We also suggest further observational study to confirm the results.

\section{Introduction}

Rheumatoid arthritis (RA) is one of the most prevalent chronic inflammatory diseases with the incidence of $0.5 \%$ to $1 \%$. It primarily involves joints, but it could affect all other parts of body [1].

The pathogenesis of RA is not fully understood, but it seems that oxidative process and loss of antioxidant defense are important in the inflammatory process [2, 3]. Migration of neutrophils and other leukocytes from blood vessels to the inflammatory area leads to increased secretion of oxidative substances and plays a key role in the progression of the RA inflammation $[4,5]$. 
Malondialdehyde (MDA) and oxidized Low-Density Lipoproteins (ox.LDL) are two of the most measurable products of oxidative process and high levels of them in RA patients is confirmed in several studies [6, 7]. On the other hand, various antioxidant systems such as Glutathione Peroxide (GSH-Px), Superoxide Dismutase (SOD) and Catalase (CAT) are also active in RA patients. SOD is the first line of defense against reactive oxygen species (ROS), catalyzes the dismutation of the superoxide anion into hydrogen peroxide. CAT, in the next step, transforms hydrogen peroxide into $\mathrm{H} 2 \mathrm{O}$ and 02 . Moreover, GSH-Px as a selenoprotein, oxidizes glutathione and reduces lipidic or nonlipidic hydroperoxides as well as $\mathrm{H} 2 \mathrm{O} 2$ [8].

The inflammatory process in the synovial compartment is regulated by a complex cytokine and chemokine network; tumor necrosis factor (TNF), interleukin (IL) 6, and probably granulocyte-monocyte colony stimulating factor are essential for it [9]. Cytokines and chemokines lead to the induction or aggravation of the inflammatory response by activating endothelial cells and attracting immune cells to accumulate within the synovial compartment.

There are so many inconsistencies regarding the role of these biomarkers in RA pathogenesis and also their relationship to RA severity. Furthermore, the correlation between inflammatory markers such as TNF$a$, IL1 $\beta$ and IL 6 with the oxidative process has not been adequately addressed. We designed this study to evaluate the level of oxidant and antioxidant and inflammatory markers in RA patients and their effects on disease activity.

\section{Materials And Methods}

\section{Materials}

Activity assay kits of GSH-Px (D-89075), SOD (K335-100) and Catalase (D-89075) from Biocore Diagnosik Ulm GmbH Co (Germany), BioVision Co (USA) and Biocore Diagnosik Ulm GmbH Co (Germany), respectively. Quantity assay kits of ox.LDL (10-1143-01), IL1 $\beta$ (850.006.048) and IL6 (860.020.048, 860.020.096, 860.020.192) from Mercodia Co (Sweden), DIACLONE Co (France), DIACLONE Co (France) and DIACLONE Co (France), respectively. Quantity assay kits of MDA (10009055) and TNF-a (DTA00C) from Cayman Co (USA) and R\&D SYSTEMS Co (USA), respectively.

\section{Methods}

\section{Samples}

$10 \mathrm{ml}$ of venous blood sample of each patient was drawn in the hospital lab. Then, each sample was mixed with anticoagulant ethylenediaminetetraacetic acid. The blood sample was centrifuged at $2500 \times$ $\mathrm{g}$ for $10 \mathrm{~min}$, and the serum was then separated and aliquoted into tubes. Samples were stored at $-70^{\circ} \mathrm{C}$ until assayed.

\section{Measuring markers}


The serum level of MDA was assessed by Colorimetric method and Cayman kit, USA). Microplate reader instrument (Sunrise model) (Teacan Co, Austria) was applied to determine mentioned parameter.

\section{ox.LDL}

Quantity level of ox.LDL in rat's serum was determined by ELISA method and Mercodia kit (Sweden). Mindray ELISA reader apparatus (MR-96A model, Germany) was used to measure related amounts.

\section{CAT}

Catalase activity of patient's serum was assessed by activity assay kit of Biocore Diagnostik Ulm GmbH Co (Germany) and colorimetric technique. Microplate reader instrument (Sunrise model) (Teacan Co, Austria) was applied to determine mentioned parameter.

\section{GSH-Px}

Glutathione peroxidase activity of patient's serum was determined by activity assay kit of Biocore Diagnostik Ulm GmbH Co (Germany) and colorimetric technique. Microplate reader instrument (Sunrise model) (Teacan Co, Austria) was applied to determine mentioned parameter.

\section{SOD}

Superoxide Dismutase activity of patient's serum was determined by activity assay kit of BioVision Co (USA) and colorimetric technique. Microplate reader instrument (Sunrise model) (Teacan Co, Austria) was applied to determine mentioned parameter.

\section{Interleukins}

The serum levels of IL1 $\beta$ and IL 6 were assessed by ELISA method and immunoenzymometric assay (DIACLONE kit, France). All measurements were done using a Mindray ELISA reader instrument (MR-96A model, Germany).

\section{TNF-a}

The serum levels of TNF-a were assessed by ELISA method and immunoenzymometric assay (R\&D SYSTEMS kit, USA). All measurements were done using a Mindray ELISA reader instrument (MR-96A model, Germany).

\section{Study population}

43 RA patients who referred to the rheumatology clinic of Imam Khomeini Hospital complex in 2017 were chosen as a case group. All of RA patients were diagnosed based on American College of Rheumatology criteria [10]. 43 healthy people who were matched with the case group were selected as a control group. The exclusion criteria were smoking (smoking longer than past 5 years), alcohol intake (drinking longer than past 12 months), using narcotics (any type of narcotic drugs at any frequency anytime), 
hypertension, diabetes mellitus, hypothyroidism, hyperthyroidism, and any other form of inflammatory arthritis except RA, receiving alternative and complementary treatments such as Ayurveda, homeopathy, and siddha.

This study was approved by ethical committee of Tehran University of Medical sciences and all participants completed the informed consent form.

\section{Data collection}

An expert rheumatologist discussed the study to the patients and questionnaires including demographic factors, disease activity Index (DAS28) and Global Health assessment were completed. Both cases and control groups were referred to the central endocrinology lab located in Imam Khomeini Hospital to evaluate the levels of oxidants, antioxidants, and other inflammatory factors. We used DAS28 to evaluate RA activity. Patients with DAS28<2.6 were considered in remission and above it were grouped as active RA [4].

\section{Data analysis}

The data was analyzed by SPSS, version 15.0. Values are expressed as the mean and standard deviation. The differences were assessed

\section{Results}

43 patients and 43 healthy volunteer were included in two groups of case and control study. The average age of patients in the case and control groups was 51.1 and 50.56 respectively with no statistically significant difference (P-Value= 0.8). 6 males and 37 females were enrolled in each group.

Significant differences were found in the average levels of MDA, ox.LDL, CAT, GSH-Px, SOD, TNF-a, IL1 $\beta$ and IL 6 between the two groups ( $p$-value $<0.001$ ). MDA, ox.LDL were higher in the case group. The average of antioxidants levels (CAT, GSH-Px and SOD) were higher in the control group than the case group. Also we noticed that levels of inflammatory cytokines such as TNF- $\alpha$, IL $1 \beta$ and IL6, were higher in the patients (table 1).

According to DAS28, 9 patients $(20.9 \%)$ were in remission, and others had an active phase of the disease (table 2). No significant differences were found in the average levels of MDA, ox.LDL, CAT, GSH-Px, SOD, also inflammatory factors in these subgroups based on DAS28 $(p>0.05)$.

\section{Discussion}

Rheumatoid arthritis is a systemic disorder characterized by chronic inflammation in the body [11]. It affects women more than men and is more common in ages over 35 years [11]. Rheumatoid arthritis is a complex disease with unknown pathophysiology, however it is obvious that tissue damages and inflammation mostly in the joints lead to arthritis [11]. In RA, the polymorphonuclear leukocytes are 
activated, and ROS are excessively generated. Studies have shown these ephemeral molecules have an important role in the progression of RA [6].

As we showed before, MDA level as an oxidant marker was higher in the case than the control group and this is congruent with several studies $[6,7,12-16]$. We believe that MDA is one of the substances produced in the lipid peroxidation process and important material to control the function of cell membrane enzymes and increase the permeability of the membrane which leads to cell destruction, as a result it contributes an important role in pathogenesis of RA. More likely it can be used as a predictor of RA development.

We found out the same result for another oxidant marker which was ox.LDL (table 1). The average level of this biomarker was higher in the patients group than controls and also in patients with active disease than remission. According to our study, it appears that these biomarkers are essential for pathogenesis and development of RA.

We also showed decreased antioxidant activity in RA patients which referred to lack of antioxidant defense in RA patients (table 1), most of the reports showed low level of GSH-Px in RA patients as we found in our study $[14,15,18,19]$ but it has been reported to be high in other studies $[5,17]$. The reduction of GSH-Px activity in RA patients happens during detoxification process because of high levels of ROS especially hydrogen peroxide. It is associated with saturation of enzymatic antioxidant systems and enzymatic inhibition. Lower level of this enzyme in patients group could happen due to severity of oxidative process [5]. Moreover, as we showed, low level of CAT in RA patients has been reported because of ROS attack $[6,14]$. On the other hand there are several controversies about SOD level in RA patients $[16,20]$ and it has been observed to be low in RA patients the same as our finding $[14,21,22]$. As a result, we suggest doing more studies to establish a definitive role of these markers in RA pathophysiology. On the other hand, higher amounts of inflammatory cytokines such as TNF-a, IL1 $\beta$ and IL6 in the case group (table 1) confirms the relationship between oxidative stress and inflammation like another study [6].

We used DAS28 criteria to investigate the effect of inflammatory biomarkers in RA severity. However, we observed that levels of oxidants were higher in active group and also antioxidants were lower remission group but we couldn't find any statistically significant differences between two groups. As a result, it seems that severity of RA is not affected by the oxidants and antioxidants activity and therefore cannot be used as a predictor of RA severity. The results of our study were incongruent with other studies [14, 23]. According to this findings, we recommend to do more observational studies with larger sample size to establish more reliable results.

\section{Conclusion}

Among our investigated biomarkers, we concluded that these biomarkers are involved in the pathogenesis and development of RA by forming an inflammatory process. Therefore, effective treatment which targets these markers could be an effective method to subside the inflammation. Based on our 
findings, it seems that the oxidative and inflammatory process did not have any effect on the severity of the disease. We suggest a further interventional study to confirm the results.

\section{Declarations}

This study was granted by Tehran University of Medical sciences with registration number: 95-02-3032343

\section{Conflict of interest}

The authors declare no conflict of interest.

\section{References}

1. Smolen, J.S., D. Aletaha, and I.B. Mclnnes, Rheumatoid arthritis. The Lancet, 2016. 388(10055): p. 2023-2038.

2. Quinonez-Flores, C.M., et al., Oxidative Stress Relevance in the Pathogenesis of the Rheumatoid Arthritis: A Systematic Review. Biomed Res Int, 2016. 2016: p. 6097417.

3. Halliwell, B. and J.M. Gutteridge, Role of free radicals and catalytic metal ions in human disease: an overview. Methods Enzymol, 1990. 186: p. 1-85.

4. Datta, S., et al., Correlation of oxidant status with oxidative tissue damage in patients with rheumatoid arthritis. Clin Rheumatol, 2014. 33(11): p. 1557-64.

5. Kerimova, A.A., et al., Antioxidant enzymes; possible mechanism of gold compound treatment in rheumatoid arthritis. Pathophysiology, 2000. 7(3): p. 209-213.

6. Sarban, S., et al., Plasma total antioxidant capacity, lipid peroxidation, and erythrocyte antioxidant enzyme activities in patients with rheumatoid arthritis and osteoarthritis. Clin Biochem, 2005. 38(11): p. 981-6.

7. Ozgunes, H., H. Gurer, and S. Tuncer, Correlation between plasma malondialdehyde and ceruloplasmin activity values in rheumatoid arthritis. Clin Biochem, 1995. 28(2): p. 193-4.

8. Kumar, V., et al., Antioxidant enzymes in rheumatoid arthritis. J Arthritis, 2016. 5(206): p. 2.

9. Feldmann, M. and S.R.N.J.I.r. Maini, Role of cytokines in rheumatoid arthritis: an education in pathophysiology and therapeutics. 2008. 223(1): p. 7-19.

10. Arnett, F.C., et al., The American Rheumatism Association 1987 revised criteria for the classification of rheumatoid arthritis. Arthritis Rheum, 1988. 31(3): p. 315-24.

11. Firestein G, R.A., Kelley's text book of Rhumatology, Harris E, Budd R, Firestein G. Pennsylvania: Elsevier \& Saunders: 2005.

12. Gambhir, J.K., P. Lali, and A.K. Jain, Correlation between blood antioxidant levels and lipid peroxidation in rheumatoid arthritis. Clin Biochem, 1997. 30(4): p. 351-5. 
13. Lunec, J., et al., Free-radical oxidation (peroxidation) products in serum and synovial fluid in rheumatoid arthritis. J Rheumatol, 1981. 8(2): p. 233-45.

14. El-barbary, A.M., et al., Assessment of lipid peroxidation and antioxidant status in rheumatoid arthritis and osteoarthritis patients. The Egyptian Rheumatologist, 2011. 33(4): p. 179-185.

15. Karatas, F., et al., Antioxidant status \& lipid peroxidation in patients with rheumatoid arthritis. Indian Journal of Medical Research, 2003. 118: p. 178-181.

16. Vyas, S., H. Sharma, and V. RK, ROLE OF MALONDIALDEHYDE IN THE SERUM OF RHEUMATOID ARTHRITIS AND OSTEOARTHRITIS. Journal of Postgraduate Medical Institute (Peshawar-Pakistan), 2016. 30(1).

17. Olivieri, O., et al., Red blood cell susceptibility to lipid peroxidation, membrane lipid composition and antioxidant enzymes in patients with rheumatoid arthritis. J Rheumatol, 1991. 18(8): p. 1263-4.

18. Taysi, S., et al., Lipid peroxidation, some extracellular antioxidants, and antioxidant enzymes in serum of patients with rheumatoid arthritis. Rheumatol Int, 2002. 21(5): p. 200-4.

19. Ashour, M., et al., Antioxidant status in children with juvenile rheumatoid arthritis (JRA) living in Cairo, Egypt. Int J Food Sci Nutr, 2000. 51(2): p. 85-90.

20. Mazzetti, I., et al., Serum copper/zinc superoxide dismutase levels in patients with rheumatoid arthritis. Int J Clin Lab Res, 1996. 26(4): p. 245-9.

21. Karatas, F., et al., Antioxidant status \& lipid peroxidation in patients with rheumatoid arthritis. Indian J Med Res, 2003. 118: p. 178-81.

22. Banford, J.C., et al., Serum copper and erythrocyte superoxide dismutase in rheumatoid arthritis. Ann Rheum Dis, 1982. 41(5): p. 458-62.

23. Patil, A.B., et al., Antioxidant gap and lipid peroxidation in patients with rheumatoid arthritis: Relationship to disease manifestations and activity. Asian Pacific Journal of Tropical Disease, 2012. 2: p. S592-S595.

\section{Tables}

Table 1: Comparison of oxidant and antioxidant status between the case and control group. 


\begin{tabular}{|c|c|c|c|c|}
\hline & Groups & Mean & Standard deviation & P-value \\
\hline MDA & Case & 3.21 & 0.35 & 0.001 \\
\hline$(\mu \mathrm{M} / \mathrm{ml})$ & Control & 2.74 & 0.37 & \\
\hline ox.LDL & Case & 17.13 & 0.90 & 0.001 \\
\hline$(\mathrm{mU} / \mathrm{ml})$ & Control & 14.43 & 1.17 & \\
\hline CAT & Case & 2.15 & 0.31 & 0.001 \\
\hline (U/ml) & Control & 2.48 & 0.36 & \\
\hline GSH-PX & Case & 84.27 & 6.79 & 0.001 \\
\hline (U/ml) & Control & 90.10 & 7.06 & \\
\hline SOD & Case & 3.93 & 0.41 & 0.001 \\
\hline (U/ml) & Control & 4.34 & 0.35 & \\
\hline TNF- $a$ & Case & 1139.39 & 73.01 & 0.001 \\
\hline (pg/ml) & Control & 514.32 & 44.80 & \\
\hline IL1 $\beta$ & Case & 644.06 & 68.54 & 0.001 \\
\hline (pg/ml) & Control & 410.02 & 44.58 & \\
\hline IL6 & Case & 612.69 & 105.90 & 0.001 \\
\hline$(\mathrm{pg} / \mathrm{ml})$ & Control & 406.60 & 48.55 & \\
\hline
\end{tabular}

MDA: Malondialdehyde, ox.LDL: oxidized Low-Density Lipoproteins, CAT: Catalase, GSH-Px: Glutathione Peroxide, SOD: Superoxide Dismutase, TNF: Tumor Necrosis Factor, IL: Interleukin

Table 2: Comparison of oxidant and antioxidant status between two groups of patients according to DAS28, remission and active group.

\begin{tabular}{|c|c|c|c|c|c|c|c|c|c|}
\hline \multicolumn{2}{|l|}{ DAS28 } & MDA & Ox.LDL & CAT & GSH-PX & SOD & TNF-a & IL1 $\beta$ & IL6 \\
\hline & & $(\mu \mathrm{M} / \mathrm{ml})$ & $(\mathrm{mU} / \mathrm{ml})$ & $(\mathrm{U} / \mathrm{ml})$ & & $(\mathrm{U} / \mathrm{ml})$ & (pg/ml) & $(\mathrm{pg} / \mathrm{ml})$ & $(\mathrm{pg} / \mathrm{ml})$ \\
\hline \multirow[t]{2}{*}{ Remission } & mean & 14.37 & 2.40 & 91.66 & 4.35 & 515.74 & 404.48 & 405.17 & 14.37 \\
\hline & & 1.10 & 0.34 & 7.16 & 0.34 & 44.78 & 42.01 & 49.59 & 1.10 \\
\hline \multirow[t]{2}{*}{ Active } & mean & 14.51 & 2.57 & 88.32 & 4.34 & 512.70 & 416.40 & 408.25 & 14.51 \\
\hline & SD & 1.26 & 0.37 & 6.75 & 0.37 & 45.87 & 46.93 & 48.70 & 1.26 \\
\hline P-Value & & 0.6 & 0.7 & 0.1 & 0.1 & 0.9 & 0.9 & 0.3 & 0.8 \\
\hline
\end{tabular}


DAS: disease activity score, MDA: Malondialdehyde, ox.LDL: oxidized Low-Density Lipoproteins, CAT: Catalase, GSH-Px: Glutathione Peroxide, SOD: Superoxide Dismutase, TNF: Tumor Necrosis Factor, IL: Interleukin, SD: Standard deviation 